\title{
P300 Asymmetries in Schizophrenia Revisited With Reference-Independent Methods
}

\author{
Werner Konrad Strik, Thomas Dierks, Ernst Franzek, Gerald Stöber, and \\ Konrad Maurer
}

Received May 3. 1993; revised version received October 5, 1993; accepted November 19. 1993.

\begin{abstract}
Evidence of hemispheric asymmetries in schizophrenia has been reported from different research areas. Asymmetries in evoked potential P300 topography are still controversial because of inconsistent findings. In the present study, previous results of abnormal lateralization of P300 were replicated in stabilized residual schizophrenic patients. Auditory P300 was recorded during an oddball task in which subjects detected rare target stimuli. Schizophrenic patients had the $\mathbf{P} 300$ peak shifted to the right hemisphere and differed significantly from age- and sex-matched normal control subjects who had left-lateralized P300 peaks. A comparison of different methods of assessment and analysis of the topographical features of the P300 electric fields showed that the extraction of reference-independent descriptors of P300 topography is a reliable and sensitive method for statistical handling of the maps. The results suggest left hemispheric dysfunction during cognitive tasks in a subgroup of schizophrenic patients. Inconsistencies between previous studies are likely to be due to heterogeneous patient groups, which may have included patients in an acute schizophrenic episode or patients in clinical remission. Investigation of the clinical meaning of P300 alterations requires careful psychopathological definition of the patient groups.
\end{abstract}

Key Words. Laterality, evoked potentials, electroencephalography.

Results from different research areas indicate asymmetries of brain function and morphology in schizophrenic patients (Jakob and Beckmann, 1986; Posner et al., 1988; Crow et al., 1989; Early et al., 1989; Roemer and Shagass, 1990; Rossi et al., 1990; Falkai et al., 1992; Min and Oh, 1992). Most of the studies suggest left hemispheric dysfunction and invoke the frontal, parietal, or temporal lobe-in particular, the medial temporal lobe and the hippocampus (Flor-Henry, 1983; Luchins, 1990). There are still controversies, however, due to inconsistent findings between, and to the large variance within, different patient populations.

\footnotetext{
Werner Konrad Strik, M.D., is Psychiatrist and Head, Laboratory of Clinical Neurophysiology, Department of Psychiatry, University Hospital of Würzburg, Germany. Thomas Dierks, M.D., is Head, Laboratory of Neurophysiology, Department of Psychiatry, University Hospital of Frankfurt, Germany. Gerald Stöber, M.D.. is Clinical and Research Assistant, Department of Psychiatry, University Hospital of Würzburg. Germany, Ernst Franzek, M.D., is Psychiatrist and Assistant Medical Director, Department of Psychiatry, University Hospital of Würzburg, Germany. Konrad Maurer, M.D., is Professor of Psychiatry and Director, Department of Psychiatry, University Hospital of Frankfurt. Germany. (Reprint requests to Dr. W.K. Strik, Laboratory of Clinical Neurophysiology, Dept. Psychiatry, University Hospital Würzburg, 97080 Würzburg, Germany.)
}

Elsevier Science Ireland Ltd. 
In neurophysiology, there is particular interest in the P300 component of event related potentials. This component is recorded during discriminative information processing and is supposed to be generated in hippocampal-parahippocampal areas with some contribution of the frontal lobe, the amygdala, and the inferior parietal lobe (Wood and McCarthy, 1985; Halgren et al., 1986; Smith et al., 1990). The electrical P300 field must be considered to be the sum of these widespread neuronal activities related to the experimental task. Since schizophrenia is thought to involve dysfunction in some of these areas, P300 parameters such as field strength, latency, and topography are therefore relevant for the study of schizophrenic disorders.

On the basis of knowledge about the eliciting paradigms and the neural generators of the P300 component, functional abnormalities in cognitive processing are expected to result in alterations of P300 amplitude and latency, while interhemispheric functional asymmetries of the involved brain structures should be detectable in the form of an asymmetrical distribution of the P300 electrical field.

A wide literature has documented reduced P300 amplitudes in schizophrenic patients (Roth et al., 1972, 1980; Brecher and Begleiter, 1983; Pritchard, 1986). Multichannel recordings, on the other hand, have shown reduced amplitudes at left temporal areas (Morstyn et al., 1983). This finding has been replicated by the same group (Faux et al., 1990; McCarley et al., 1993) but was not confirmed by independent investigators (Pfefferbaum et al., 1989).

Strik et al. (1993), who applied reference-independent assessment of topography, reported a trend for right lateralization of the P300 peak in a group of remitted and residual $D S M-I I I-R$ schizophrenic patients. After psychopathological subclassification of the patients into cycloid psychoses (fully remitted) and noncycloid psychoses (who were in a residual state at the time of the examination), the right lateralization of the P300 peaks and P300 minima of the residual patients was found to be significant with respect to control subjects, whereas cycloid psychoses did not differ from control subjects. Since amplitudes were significantly lower in the residual group than in control subjects, it appeared that the two parameters (amplitudes and topography) might be associated and reflect a reduction of left-hemispheric activity.

The present study was designed to confirm P300 topographical asymmetries in a new patient sample. On the basis of the previous study (Strik et al., 1993), P300 peaks and P300 minima were expected to be right lateralized in a selected group of stabilized residual schizophrenic patients.

\section{Methods}

Subjects. The present patient group consisted of 25 inpatients and outpatients from the Psychiatric University Hospital of Würzburg and the Psychiatric Hospital of Lohr. To match the clinical features of the patients in the previous study (Strik et al., 1993), only patients in a residual schizophrenic state were included. Fully remitted patients and patients in an acute psychotic episode were excluded from the study. Accordingly, inclusion criteria were as follows: DSM-III-R diagnosis of residual schizophrenia (American Psychiatric Association, 1987) assigned independently by two senior psychiatrists; stabilized clinical condition as defined by stable psychopathology (judged independently by two psychiatrists) and unchanged medication for a minimum of 2 weeks; no concomitant medical or neurological diseases, alcoholism, or drug abuse. Three of the 25 schizophrenic patients had to be excluded 
from analysis because of artifacts or an excessive counting error in the neurophysiological investigation. The values reported below refer to the remaining 22 patients ( 6 women, 16 men).

The mean dose of neuroleptic medication was $331 \mathrm{mg}(\mathrm{SD}=262)$ in chlorpromazine equivalents; none of the patients received lithium, carbamazepine, benzodiazepines, or antidepressants. Two psychiatrists independently judged that the patients had achieved the best possible equilibrium between residual symptoms, medication, and social functioning. The mean total score on the Brief Psychiatric Rating Scale (Overall and Gorham, 1962) was 36.23 $(S D=7.21)$, and the mean global score on the Scale for the Assessment of Negative Symptoms (Andreasen, 1983) was $12.7(\mathrm{SD}=6.1$ ). Scores on both measures were similar to those of the previous hypothesis-building group (Strik et al., 1993). All of the patients participated in long-term social rehabilitation programs. The mean age was 35.4 (SD = II.4; range $=19-67$ ) years. With the exception of one ambidextrous patient, all patients were right-handed.

Twenty-two control subjects ( 6 women, 16 men) were recruited from the hospital staff and their relatives, and were age- and sex-matched with the patients. The mean age was $31.6(\mathrm{SD}=$ 12.6; range $=20-71$ ) years and did not differ significantly from that of the schizophrenic patients $(t=1.06, \mathrm{NS})$. All of the control subjects were right-handed, and none had a history of neurological disorder, psychiatric disorder, or drug abuse.

Neurophysiological Recordings. P300 was elicited with a standard oddball paradigm. Subjects heard binaural tones presented through headphones (tone burst, $50 \mathrm{~ms}$; rise and fall time, $10 \mathrm{~ms})$. A frequent tone of $1000 \mathrm{~Hz}$ had to be ignored, and a rare tone $(2000 \mathrm{~Hz}) \mathrm{had}$ to be silently counted by the subject. The rate of stimulus presentation was $0.5 /$ second; $20 \%$ of the stimuli were targets. The subjects had to report the number of rare tones they had heard at the end of the trial in order to control performance. The recordings were accepted if the counting error did not exceed $\pm 10 \%$.

Recordings were obtained from 20 electrodes placed according to the international 10-20 system (see Fig. 1); Fpz was skipped and later interpolated by the recording system (BioLogic Brain Atlas). Recording references were linked mastoids with compensating resistors of 10 kOhm each. Automated artifact rejection supplied by the system based on an amplitude window was applied; data were filtered with a bandpass from 0.3 to $70 \mathrm{~Hz}$, and the digitization rate was $250 \mathrm{~Hz}$. As a final control, the recordings were visually inspected for artifacts.

Parameters and Statistics. All P300 features were determined in the time window from 270-470 ms. Amplitudes were assessed with two methods. First, using a reference-independent procedure, the latency of maximal Global Field Power (Lehmann and Skrandies, 1980), i.e., the time point of maximal field strength within the time window was determined. The map at this latency was further analyzed. P300 amplitudes were defined as the difference of the maximal and the minimal potential value within the entire map, excluding the reference (Lehmann, 1987; Dierks and Maurer, 1990). This amplitude measure will be referred to as the reference-independent amplitude.

Second, following traditional conventions, the midline parietal electrode $(\mathrm{Pz})$ was chosen as the active electrode. Latencies were determined at the moment of the individual peaks in this channel. Pz amplitudes vs. linked mastoids after subtraction of a 220-ms prestimulus baseline were determined at the latency of the grand average $\mathrm{Pz}$ wave shape $(368 \mathrm{~ms})$. These referencedependent procedures were applied to allow comparisons of the major P300 parameters with results from the literature.

The topographical configuration of the P300 electric fields was globally quantified based on topographical descriptors; that is, on the locations of the extreme (highest and lowest) potential values of the P300 map. For this purpose, the electrode array was projected onto the cross-points of a rectangular grid, and coordinate values from 1 to 5 were assigned for each electrode row in the sagittal and in the coronal direction (see Fig. 1). The location of each electrode site is thus quantified by two coordinates (e.g., the Pz electrode by 4 in the anteriorposterior and by 3 in the left-right direction). Again, the map at the individual latency of 
Fig. 1. Electrode array and coordinate values for electrode rows 1.5 in the sagittal and coronal direction

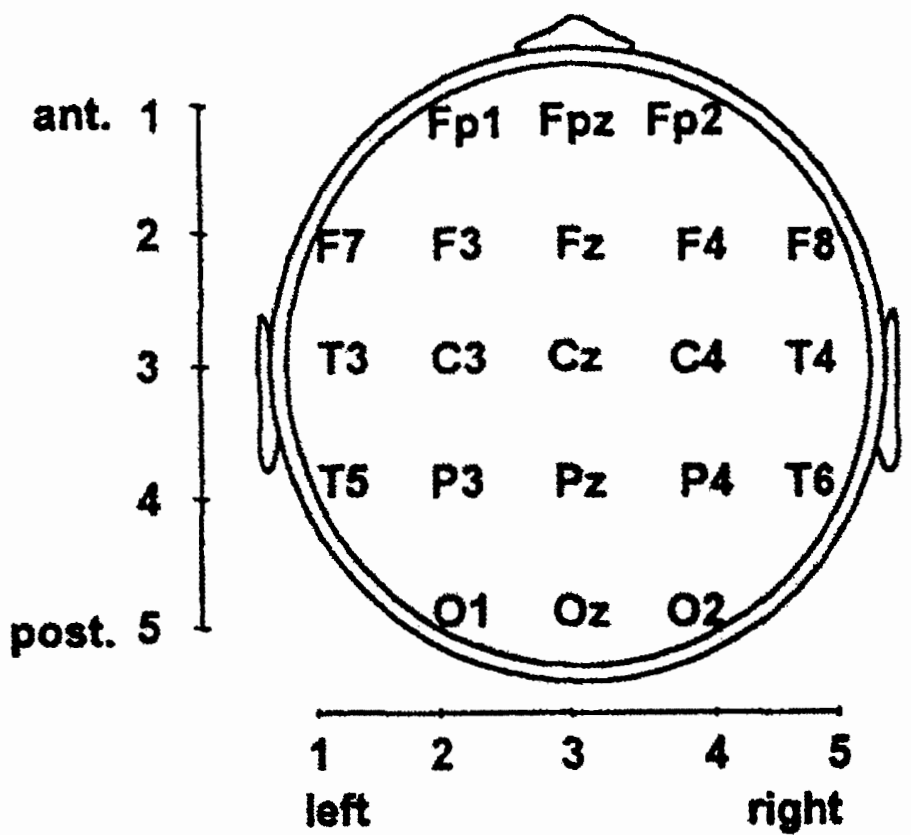

The coordinate values are used to quantify the locations of the topographical descriptors of the P300 electric field configurations.

maximal Global Field Power in the time window from $270-470 \mathrm{~ms}$ was analyzed. The coordinates of the electrodes with the extreme potential values (peak and minimum) of this map were used as topographical P300 descriptors. Since no interpolated values were used for analysis, the locations of the extreme values of the maps are identical with the respective electrode positions. This method extracts pure topographical features independent of the reference. Statistical comparisons of map configurations between subjects can therefore be computed independent of absolute potential values (Lehmann, 1971, 1987).

Confirmatory statistics. The differences of P300 amplitudes and latencies between groups were tested with paired $t$ tests. Topographical differences of the P300 maps were tested between patients and control subjects with Wilcoxon's matched pairs tests on the locations of the topographical P300 descriptors; that is, the locations of the peak and of the minimum in the sagittal (anterior-posterior) and coronal (left-right) direction.

Post hoc tests. All correlations with the locations of the P300 peaks were performed with Spearman's rank correlation analysis; for correlations with amplitudes, we computed Pearson's $\rho$. For illustration ( $t$ map, Fig. 6 bottom), single $t$ tests were computed for each electrode between patients and control subjects at the individual P300 peaks.

Because P300 asymmetries are controversial, we computed additional statistical tests to allow comparisons between the methods of analysis applied by others (Morstyn et al., 1983; Pfefferbaum et al., 1989, 1991; Faux et al., 1990). For this purpose, we performed (1) MannWhitney $U$ tests for the amplitude ratios at preselected electrode sites of the left hemisphere and the corresponding sites of the right hemisphere (T3/T4, C3/C4); (2) a repeated measures analysis of variance (ANOVA) for the coronal row T3-C3-Cz-C4-T4; (3) a multivariate analysis of variance (MANOVA) for the same coronal row; and (4) univariate followup tests at the single electrode sites.

\section{Results}

Amplitudes. Fig. 2 shows the mean event related potential (ERP) wave shapes at the $\mathrm{Pz}$ electrode and the Global Field Power to rare tones for patients (bold) and control subjects. Fig. 3 displays the grand average wave shapes at all electrode sites. 
Fig. 2. Grand average wave shapes of the event-related potentials after target stimuli of schizophrenic patients (heavy lines) and control subjects (thin lines)
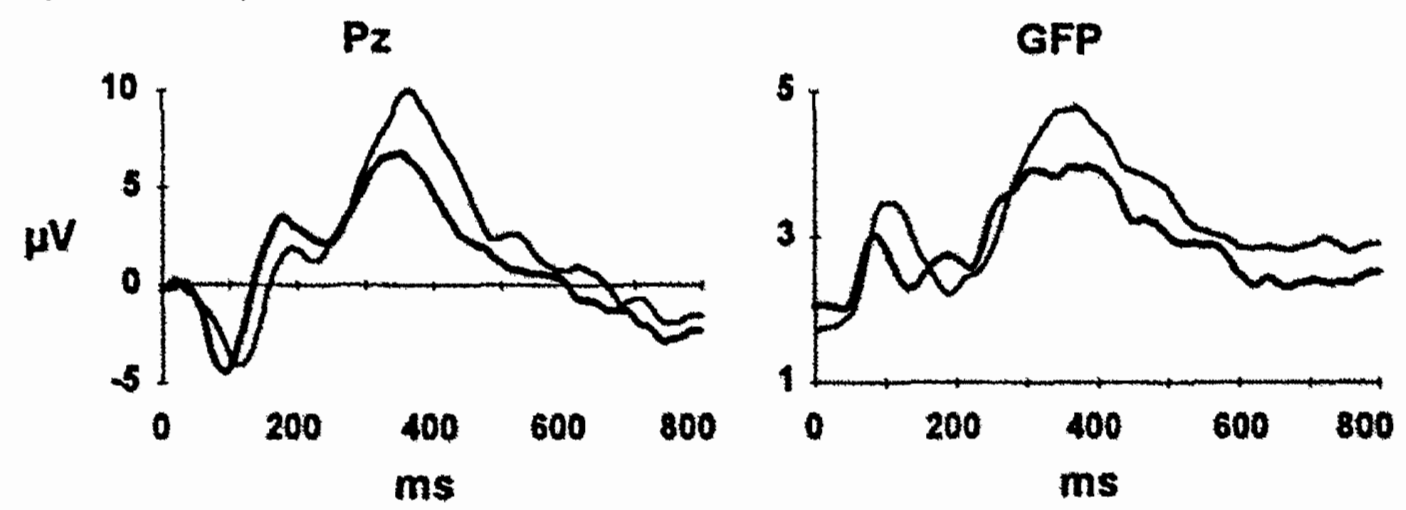

Lett: Wave shapes at the midline parietal electrode (Pz). Right: Global Field Power (spatial variance of the electrical field, GFP): schizophrenic patients have lower amplitudes at $P z$, but the GFP did not differ significantly.

Fig. 3. Grand average event-related potential (ERP) wave shapes at all electrode sites
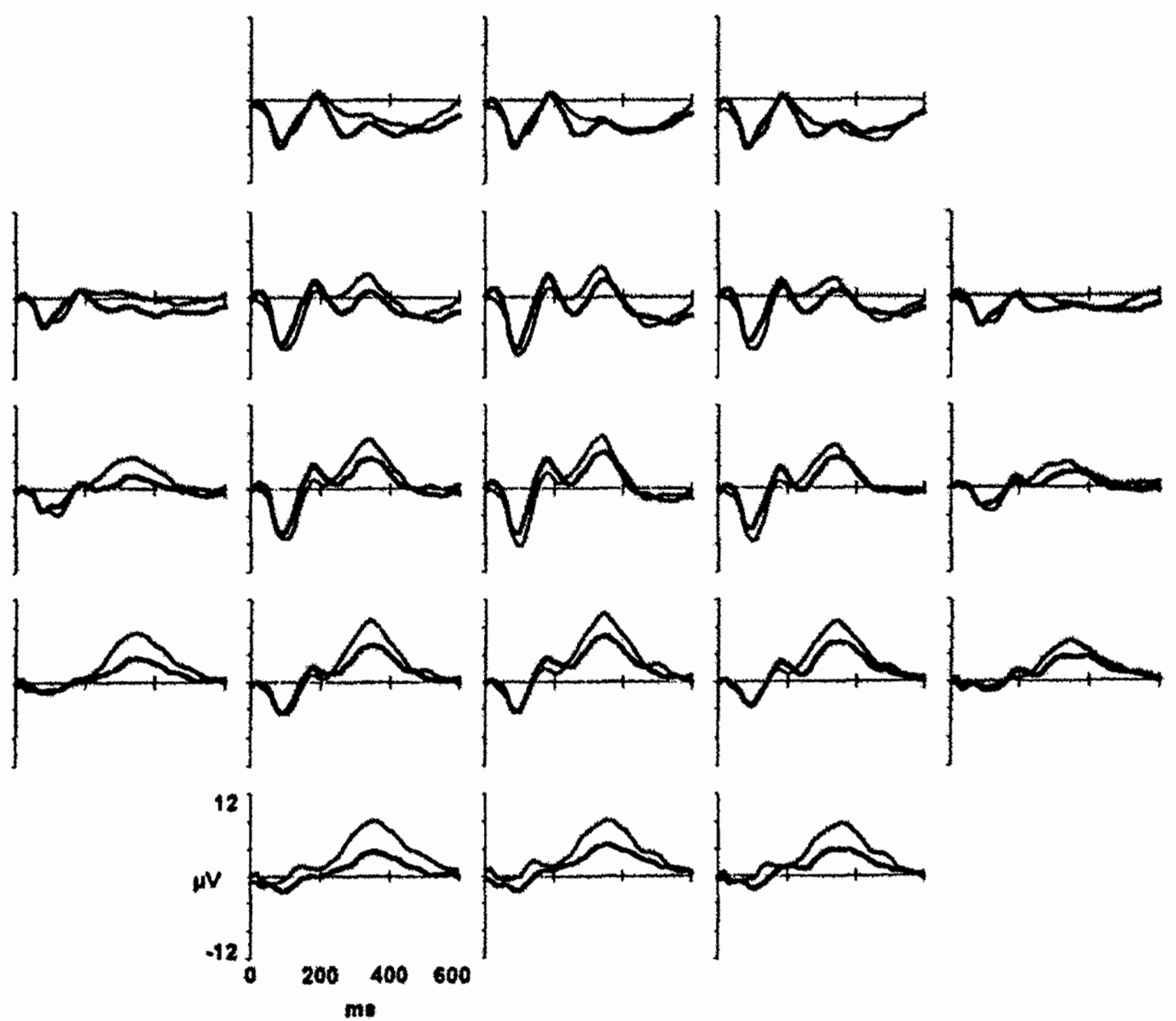

Heavy lines are ERPs of schizophrenic patients; thin lines are ERPS of control subjects. Amplifude differences in the P300 range are most pronounced in left temporal and occipital areas. 
Confirmatory statistics. Amplitudes at $\mathrm{Pz}$ at the moment of the peak of the grand average $(368 \mathrm{~ms})$ were significantly lower in the schizophrenic group compared with control subjects (Table 1). Reference-independent amplitudes, Global Field Power, and latencies assessed with both procedures did not differ between patients and control subjects, however.

Post hoc tests. To explore a possible further factor for group heterogeneity, we tested male vs. female subjects. There was a trend for the amplitudes at $\mathrm{Pz}$ to be lower ( $\mathrm{Pz}$ amplitude: $t=1.92, d f=42, p=0.07$; reference-independent amplitude: $t$ $=1.2, d f=42, \mathrm{NS}$ ) in schizophrenic males than in schizophrenic females. No such effect was found in the control group ( $\mathrm{Pz}$ amplitude: $t=1.2, d f=42$, NS; referenceindependent amplitude: $t=0.1$, NS).

Table 1. P300 amplitudes and latencies

\begin{tabular}{lrrrrrrr}
\hline & \multicolumn{2}{c}{ Schizophrenic patients } & & \multicolumn{3}{c}{ Control subjects } & \\
\cline { 2 - 3 } & Mean & SD & & Mean & SD & \\
\hline Amplitudes & & & & & & \\
Pz & 6.8 & 6.5 & & 10.0 & 3.7 & 2.19 \\
Reference independent & 17.4 & 8.4 & & 18.7 & 5.2 & 0.54 \\
GFP & 3.9 & 2.9 & & 4.9 & 1.8 & 1.54 \\
Latencles & & & & & & \\
Peak of GFP & 363.1 & 51.5 & & 354.7 & 39.4 & 0.58 \\
Peak at Pz & 363.8 & 45.0 & & 334.9 & 37.2 & 0.97 \\
\hline
\end{tabular}

Note. GFP = Global Field Power. $\mathrm{Pz}=$ midline parietal electrode.

1. Paired $t$ test: $p<0.05$.

Topography. In topographic analyses, the evoked potential data are viewed as a sequence of momentary maps. Fig. 4 plots the average locations of the maximal amplitudes of the evoked potential map sequence for the anterior-posterior and for the left-right direction for patients (bold) and control subjects. In the anteriorposterior direction, no evidence of differences between patients and control subjects can be seen over the entire time range of the ERPs (Fig. 4, left), whereas the peaks of

Fig. 4. Grand average of the locations of the maximal amplitudes of the event-related potential (ERP) map sequence in the anterior-posterior (left) and left-right direction (right)
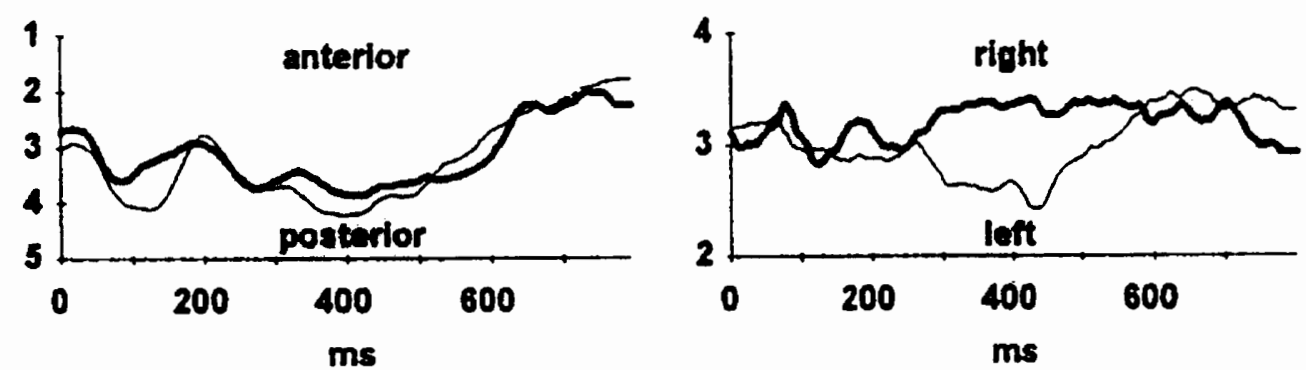

Heavy lines are the trajectories of the schizophrenic group. In the anterior-posterior direction, the $y$-axis value 1 corresponds to the frontopolar coronal row, 5 to the occipital row; in the left-right direction, 3 corresponds to the midline (see Fig. 1). In the time range of the P300 component, schizophrenic patients had the meximal amplitudes located over the right hemisphere, whereas control subjects had the peaks located over the lef hemisphere. 
the patients' ERP maps appear to be located more on the right in the time range from about 320 to $550 \mathrm{~ms}$ after stimulus onset (Fig. 4, right). Statistical tests of this and other possible topographical differences are described below.

Confirmatory statistics. The statistical tests for the differences of the descriptor locations were computed at the individual P300 latencies. As predicted by our hypothesis, the P300 peaks were located significantly more on the right side in schizophrenic patients than in control subjects. Lateralizations to the right of the P300 minimum could not be confirmed in the present sample, however. As expected, no differences were found for the locations of the extrema in the anterior-posterior direction (Table 2, Fig. 5).

Table 2. Locations of P300 topographical descriptors (peak and minimum)

\begin{tabular}{|c|c|c|c|c|c|c|c|c|}
\hline & \multicolumn{4}{|c|}{ P300 peak } & \multicolumn{4}{|c|}{ P300 minimum } \\
\hline & \multicolumn{2}{|c|}{$\begin{array}{l}\text { Left- } \\
\text { right }\end{array}$} & \multicolumn{2}{|c|}{$\begin{array}{l}\text { Anterior- } \\
\text { posterior }\end{array}$} & \multicolumn{2}{|c|}{$\begin{array}{l}\text { Left- } \\
\text { right }\end{array}$} & \multicolumn{2}{|c|}{$\begin{array}{l}\text { Anterior- } \\
\text { posterior }\end{array}$} \\
\hline & Mean & SD & Mean & SD & Mean & SD & Mean & SD \\
\hline \multicolumn{9}{|c|}{$\begin{array}{l}\text { Schizophrenic } \\
\text { patients }\end{array}$} \\
\hline $\begin{array}{l}\text { Control } \\
\text { subjects }\end{array}$ & & & & & & & & \\
\hline$(n=22)$ & 2.45 & 0.17 & 4.18 & 0.14 & 3.50 & 0.26 & 1.23 & 0.09 \\
\hline$z$ & \multirow{2}{*}{\multicolumn{2}{|c|}{3.21}} & \multirow{2}{*}{\multicolumn{2}{|c|}{0.25}} & \multicolumn{2}{|c|}{1.54} & \multicolumn{2}{|c|}{0.30} \\
\hline$p$ & & 0.001 & & NS & \multicolumn{2}{|c|}{ NS } & \multicolumn{2}{|c|}{ NS } \\
\hline
\end{tabular}

Note. Wilcoxon's matched pairs test was used.

Post hoc tests. To explore a possible further factor for group heterogeneity, we tested the locations of the P300 peak between males and females. There was a trend of the P300 peak to be more right lateralized in male schizophrenic patients compared with female patients $(z=1.80, p=0.07)$. No male-female difference was found in control subjects $(z=1.1, \mathrm{NS})$.

Multiple $t$ tests at all electrode sites were performed to construct the $t$ map in Fig. 6. Group differences were most evident in left temporal and left occipital regions.

To compare our data with previous results of other investigators, and to compare different analytic approaches, we computed additional statistics on P300 topography: The left-right amplitude ratios at the electrodes $\mathrm{T} 3 / \mathrm{T} 4$ and $\mathrm{C} 3 / \mathrm{C} 4$ were lower in schizophrenic patients than in control subjects (Table 3). A repeated measures ANOVA was performed for the coronal row T3-C3-Cz-C4-T5; the main effect (groups: $F=4.43 ; d f=1,42 ; p<0.05$ ) and the repeated measures effect (electrode: $F=24.91 ; d f=4,168 ; p<0.00001$ ) were significant. However, no effect of topography as indicated by the diagnosis $X$ electrode interaction was found with this test $(F=1.53 ; d f=4,168, \mathrm{NS})$. Finally, we performed a MANOVA with the same coronal row electrodes as dependent variables. Again, the main effect (groups) was significant (Wilk's $\lambda=0.66 ; d f=5,38 ; p=0.005$ ). The univariate followup tests 
Fig. 5. Grand average of the locations of the P300 peaks at the individual P300 latencies of schizophrenic patients $(n=22: S)$ and control subjects $(n=22:$ C)

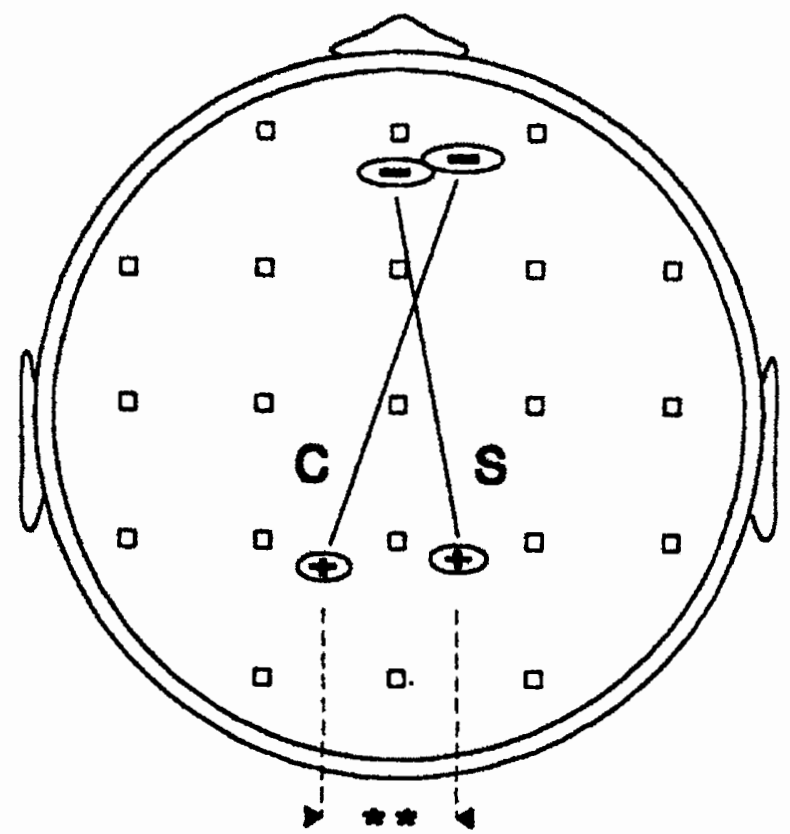

The P300 peaks are indicated by + , minima by -: ellipses indicate the standard error; ${ }^{* *} p=0.001$. The $P 300$ peaks of schizophrenic patients were located significantly more to the right side than those of the control subjects (for statistics. see Table 2).

showed significantly lower amplitudes in schizophrenic patients at the T3 electrode and, with weak probability levels, also at C3 and T4 (Table 4).

Correlations Within the Patient Group. There were no significant correlations between left-right location of the P300 peaks and dosage of medication $(\rho=-0.29$; negative sign implies an inverse correlation, i.e., higher dosages tend to be associated with more left-sided P300 peaks), or between left-right location and age $(\rho=-0.13)$. Similarly, no correlations were found between amplitudes and medication (referenceindependent: $\rho=0.19$, amplitude at the Pz electrode: $\rho=0.21$ ), and amplitudes and age (reference-independent: $\rho=-0.14$, amplitude at the $\mathrm{Pz}$ electrode: $\rho=0.02$ ).

\section{Discussion}

In the present study, lower amplitudes at the midline parietal electrode and rightlateralized peaks of the P300 electric field were found in a group of residual schizophrenic patients, replicating earlier results (Morstyn et al., 1983; Faux et al., 1990; Strik et al., 1993). Right-sided lateralization of the minimum of the P300 map (Strik et al., 1993), however, was not confirmed. In control subjects, there was a slight lateralization of the P300 peak to the left side. Single electrode comparisons showed that the amplitude differences between patients and control subjects were most pronounced in left temporal and occipital areas. No effect of medication on amplitudes or on the locations of the P300 peaks was found. It is interesting to note that the highly significant topographical difference of schizophrenic P300 cannot be 
Fig. 6. Grand average P300 maps of control subjects (top left) and schizophrenic patients (top right) and $t$-test $t$ values (bottom center) of the differences between the upper two mean maps

\section{$-11$}
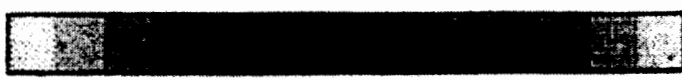

11

\section{uVolts}

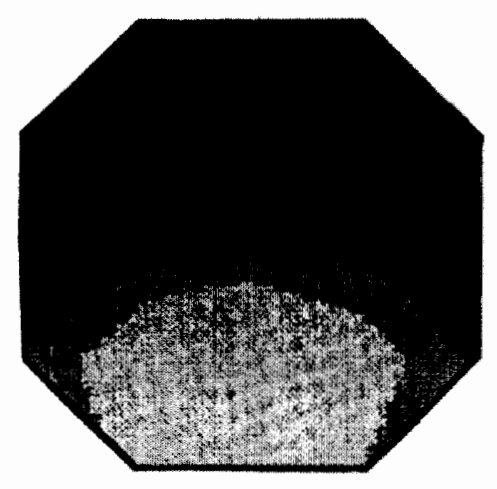

Controls

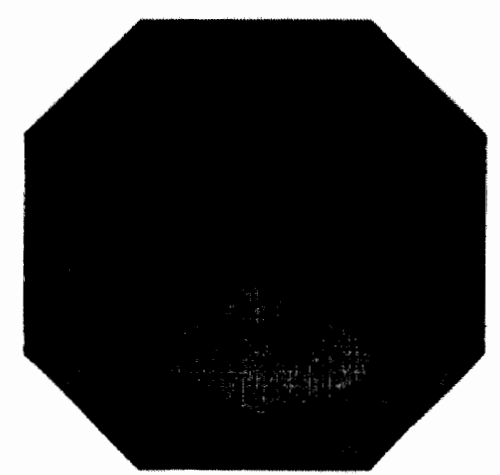

Schizophrenics

$-4$

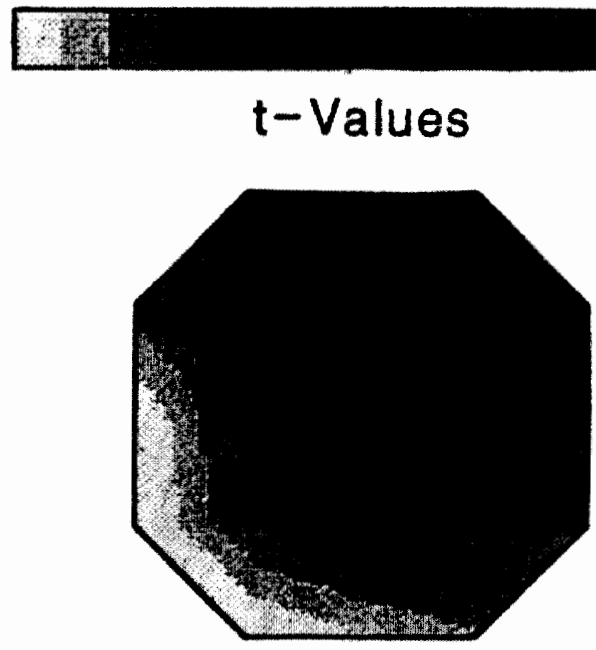

$\mathrm{t}-\mathrm{Map}$

Head seen from above, top of the map is anterior, and left is left. Blue is negative, and red is positive, referred to linked mastoids. The topographical differences between the groups cannot be seen easily in the grand average maps which are, in the schizophrenic group, dominated by the maps of some patients with high amplitudes and normal topography. The $t$-map difference is most significant in the left temporal and occipital areas. 
Table 3. Left-right anplitude ratios

\begin{tabular}{lcccc}
\hline Ratio & $\begin{array}{c}\text { Schlzophrenic } \\
\text { petionts }\end{array}$ & $\begin{array}{c}\text { Control } \\
\text { subjects }\end{array}$ & $\boldsymbol{z}$ & $\begin{array}{c}\text { 1-talled } \\
\boldsymbol{p}\end{array}$ \\
\hline T3/T4 geometric mean & 0.64 & 1.36 & & \\
Log. (geometric mean) & -0.44 & 0.31 & 1.73 & 0.04 \\
C3/C4 geometric mean & 0.99 & 1.11 & & \\
Log. (geometric mean) & -0.01 & 0.10 & 1.14 & NS \\
\hline
\end{tabular}

Note. Reported values are geometrical means and their natural logarithms. Negative values of the loge indicale amplitudes left < right; positive values indicate left > right. Mann-Whitney $U$ tests were performed.

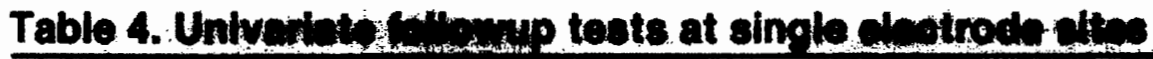

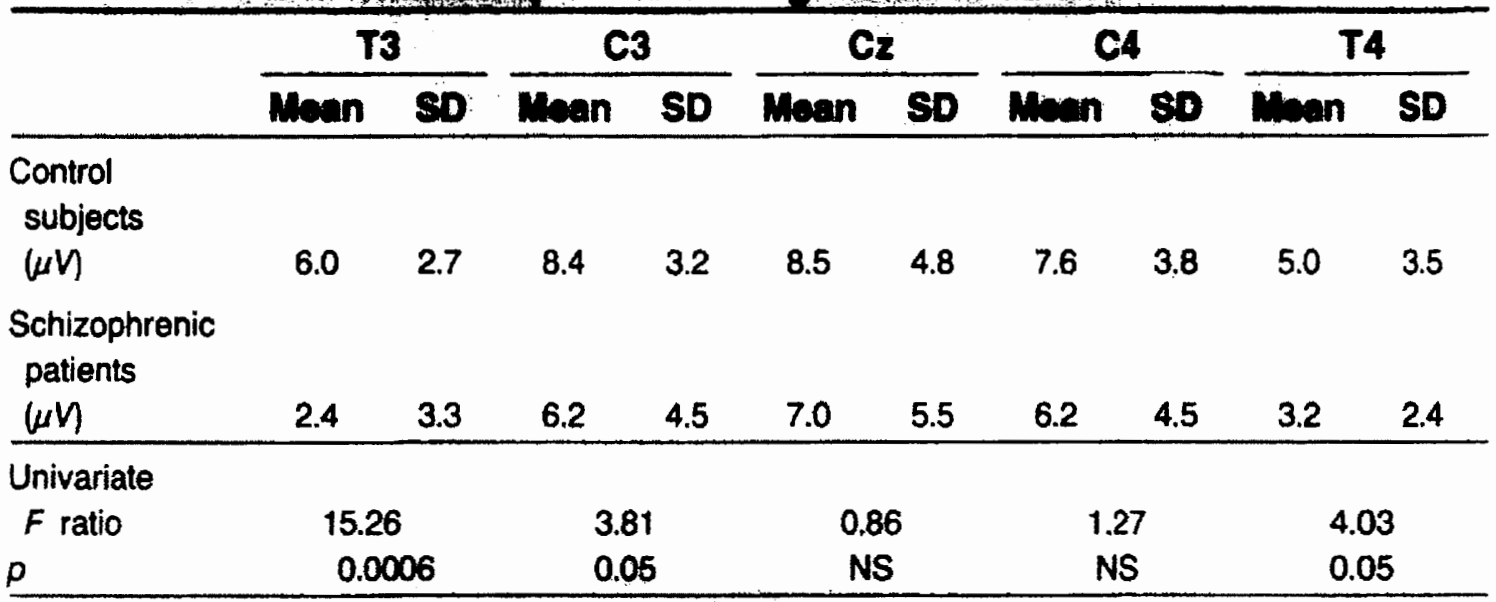

easily seen in the grand average map that is dominated by the high amplitude-normal topography maps of some patients (Fig. 6, top).

The finding of asymmetric topography comprising right hemispheric lateralization of the P300 peak and lower amplitudes at left-hemispheric electrode sites is consistent with the results of Morstyn et al. (1983) and Faux et al. (1990). Pfefferbaum et al. $(1989,1991)$, however, were not able to replicate the finding even when they applied the statistical procedure used by Faux et al. (1990).

The statistical analysis of our study was performed based on topographical descriptors; that is, the locations of the extreme values of the P300 electric field configuration as proposed by Lehmann $(1971,1987)$. This procedure allows a global assessment of the map topography with meaningful data reduction independent of the choice of the reference. On the basis of topographical descriptors, statistical handling of multichannel recordings is possible without the need for multiple tests at each electrode site (left-right P300 peak asymmetries can be investigated with a single statistical test). Furthermore, the descriptors are independent of absolute potential values that may distort topographical differences (Brandeis et al., 1987; Strik et al., 1992). The results of the present study show that this method of data reduction and statistical analysis is reliable and sensitive to detect the topographical P300 asymmetries in schizophrenic patients.

Methodological factors have been invoked to explain controversial findings of P300 topography in schizophrenia (McCarley et al., 1991). In the attempt to shed 
light on this issue, we examined our data applying the various analysis methods that have been used by the different authors. When the statistical procedure used by Faux et al. (1990) was applied, our data replicated the results of these authors. The ratios between left and right hemispheric temporal and central electrode sites were lower in the schizophrenic group than in control subjects, but the significance levels were weak. A MANOVA analysis showed significantly lower amplitudes in the coronal row T3-C3-Cz-C4-T4 in schizophrenic patients, and the followup analysis showed that amplitudes were significantly lower at the left temporal T3 electrode (Table 4).

A repeated measures ANOVA for the same coronal row, on the other hand, showed the main effect of lower amplitudes in schizophrenic patients, but it failed to reach significance in the group $\times$ electrode interactions indicative of topographical differences between the groups. This latter negative result is in principle consistent with the study of Pfefferbaum et al. (1989) and shows that the repeated measures ANOVA was not sensitive to topographical group differences.

The comparison between methods indicated that both MANOVA and repeated measures ANOVA analyses were adequate to test overall amplitude differences at multiple electrode sites. The disclosure of the topographical differences, however, was in both cases left to multiple univariate followup tests. The left-right ratios as a direct test of topographies, on the other hand, required multiple testing and yielded weak significance values in our data as in the Faux et al. study; these low significances would not withstand the $\alpha$-error correction for multiple testing. The weak significance levels may be due to interindividual differences in the sites of major asymmetry in the patient group ( $\mathrm{T} 3 / \mathrm{T} 4$ or $\mathrm{C} 3 / \mathrm{C} 4)$ which increased variance of the single ratios. We conclude that the analysis based on the topographical descriptors that we applied for confirmatory statistics was the most sensitive and straightforward method to detect global topographical differences between the groups.

The differences between methods of assessment and statistical analysis, however, probably do not account for the failure of Pfefferbaum et al. (1991) to replicate topographical assymmetries. State-dependent variations of P300 features (Duncan et al., 1987) and a lack of left-right asymmetries in completely remitted schizophrenic patients (i.e., cycloid psychosis; Strik et al., 1993) and in patients without marked reduction of the left posterior superior temporal gyrus (McCarley et al., 1993) have been demonstrated. Differences in the patient populations are therefore likely to constitute a major source of inconsistencies between studies. The successful attempt in the present study to select a more homogeneous patient group based on psychopathological features supports this view.

P300 results are frequently interpreted in terms of signal-generating structures in the brain. Interpretations in this direction should be done with extreme caution because estimates of the electric sources are ambiguous. The interpretation of the generating structure of a scalp potential as being located perpendicularly below the electrode that recorded a maximal potential is simplistic and implies the unproven assumption of a vertical generator orientation. Two basic statements, however, seem to be acceptable at the actual state of knowledge: (1) The neuronal generators are located bilaterally in the two hemispheres and typically have near-symmetric electric 
activity in healthy subjects. (2) In the absence of artifacts, different configurations of the electric field at the scalp must be generated by different patterns of neuronal activation. The second statement is mandatory within one subject, but it can be plausibly applied to group differences. Lower amplitudes and right hemispheric lateralization of the $\mathbf{P} 300$ peak (with lower potentials especially at the electrodes over left temporal areas, as again observed in the present study) accordingly might be suggested as further evidence of left hemispheric dysfunction in schizophrenic patients. This interpretation is supported by the recent article by McCarley et al. (1993), who reported reduced amplitudes at the left temporal electrode (T3) associated with magnetic resonance imaging volume reduction of the left posterior superior temporal gyrus in schizophrenia.

In conclusion, left-right asymmetries of P300 topography were confirmed in the present study. The results indicate that the psychopathological inclusion criteria of our patient group as developed in an earlier study (Strik et al., 1993)-that is, "absence of an acute episode" and "presence of residual symptoms"-were successful in identifying a more homogeneous patient population. However, there was still a considerable variability within the sample. This regards the trend of the P300 abnormalities to be more accentuated in male than in female schizophrenic patients as well as the fact that many patients had perfectly normal P300 amplitudes and P300 topographies. Further work is necessary to improve the definition of the clinical, neuropsychological, and neuropathological features associated with P300 asymmetries in schizophrenia.

\section{References}

American Psychiatric Association. DSM-III-R: Diagnostic and Statistical Manual of Mental Disorders. 3rd ed., revised. Washington, DC: American Psychiatric Press, 1987.

Andreasen, N.C. The Scale for the Assessment of Negative Symptoms. Iowa City: The University of Iowa, 1983.

Brandeis, D.; Horst, A.; and Lehmann, D. Topographic effects of attention and subjective figure perception in adaptively segmented ERP map series. In: Johnson, R., Jr.; Rohrbaugh, J.W.; and Parasuraman, R., eds. Current Trends in Event-related Potential Research. (EEG Suppl. 40) Amsterdam: Elsevier, 1987. pp. 76-80.

Brecher, M., and Begleiter, $H$. Event-related brain potentials to high-incentive stimuli in unmedicated schizophrenic patients. Biological Psychiatry, 18:661-674, 1983.

Crow, T.J.; Ball, J.; Bloom, S.R.; Brown, R.; Bruton, C.J.; Colter, N.; Frith, C.D.; Johnstone, E.C.; Owens, D.G.; and Roberts, G.W. Schizophrenia as an anomaly of development of cerebral asymmetry: A postmortem study and a proposal concerning the genetic basis of the disease. Archives of General Psychiatry, 46:1145-1150, 1989.

Dierks, T., and Maurer, K. Reference-free evaluation of auditory evoked potentials-P300 in aging and dementia. In: Dostert, P.; Riederer, P.; Strolin Benedetti, M.; and Roncucci, R., eds. Early Markers in Parkinson's and Alzheimer's Disease. Berlin: Springer, 1990. pp. 197-208. 
Duncan, C.C.; Morihisa, J.M.; Fawcet, R.W.; and Kirch, D.G. P300 in schizophrenia: State or trait marker? Psychopharmacology Bulletin, 23:497-501, 1987.

Early, T.S.; Posner, M.l.; Reiman, E.M.; and Raichle, M.E. Hyperactivity of the left striato-pallidal projection: Part I. Lower level theory. Psychiatric Development, 7:85-108, 1989.

Falkai, P.; Bogerts, B.; Greve, B.; Pfeiffer, U.; Machus, B.; Folsch-Reetz, B.; Majtenyi, C.; and Ovary, I. Loss of sylvian fissure asymmetry in schizophrenia: A quantitative post mortem study. Schizophrenia Research, 7:23-32, 1992.

Faux, S.F.; Shenton, M.E.; McCarley, R.W.; Nestor, P.G.; Marcy, B.; and Ludwig, A. Preservation of P300 event-related potential topographic asymmetries in schizophrenia with use of either linked-ear or nose reference sites. Electroencephalography and Clinical Neurophysiology, 75:378-391, 1990.

Flor-Henry, P. The Cerebral Basis of Psychopathology. Littleton, MA: Wright PSG, 1983.

Halgren, E.; Stapleton, J.M.; Smith, M.; and Altafullah, I. Generators of the human scalp P3(s). In: Cracco, Q., and Bodis-Wollner, l., eds. Evoked Potentials. New York: Alan Liss, 1986. pp. 269-284.

Jakob, H., and Beckmann, H. Prenatal developmental disturbances in the limbic allocortex in schizophrenics. Journal of Neural Transmission, 65:303-326, 1986.

Lehmann, D. Multichannel topography of human alpha EEG fields. Electroencephalography and Clinical Neurophysiology, 31:439-449, 1971.

Lehmann, D. Principles of spatial analysis. In: Gevins, A.S., and Rémond, A., eds. Methods of Analysis of Brain Electrical and Magnetic Signals. Handbook of Electroencephalography and Clinical Neurophysiology. Vol. I, revised edition. Amsterdam: Elsevier, 1987. pp. 309-354.

Lehmann, D., and Skrandies, W. Reference free identification of components of checkerboard-evoked multichannel potential fields. Electroencephalography and Clinical Neurophysiology, 48:609-621, 1980.

Luchins, D.J. A possible role of hippocampal dysfunction in schizophrenic symptomatology. Biological Psychiatry, 28:87-91, 1990.

McCarley, R.W.; Shenton, M.E.; Nestor, P.G.; and Holinger, D.P. Is there P300 asymmetry in schizophrenia? Archives of General Psychiatry, 48:380-381, 1991.

McCarley, R.W.; Shenton, M.E.; O'Donnel, B.F.; Faux, S.F.; Kikins, R.; Nestor, P.G.; and Jolesz, F.A. Auditory P300 abnormalities and left posterior superior temporal gyrus volume reduction in schizophrenia. Archives of General Psychiatry, 50:190-197, 1993

Min, S.K., and Oh, B.H. Hemispheric asymmetry in visual recognition of words and motor response in schizophrenic and depressive patients. Biological Psychiatry, 31:255-262, 1992.

Morstyn, R.; Duffy, F.H.; and McCarley, R.W. Altered P300 topography in schizophrenia. Archives of General Psychiatry, 40:729-734, 1983.

Overall, J.E., and Gorham, D.R. The Brief Psychiatric Rating Scale. Psychological Reports, 10:799-812, 1962.

Pfefferbaum, A.; Ford, J.M.; White, P.M.; and Roth, W.T. P3 in schizophrenia is affected by stimulus modality, response requirements, medication status, and negative symptoms. Archives of General Psychiatry, 46:1035-1044, 1989.

Pfefferbaum, A.; Ford, J.M.; White, P.M.; Roth, W.T.; and Mathalon, D.H. Is there P300 asymmetry in schizophrenia? In reply. Archives of General Psychiatry, 48:381-382, 1991.

Posner, M.I.; Early, T.S.; Reimann, E.; Pardo, P.J.; and Dhawan, M. Asymmetries in hemispheric controls of attention in schizophrenia. Archives of General Psychiatry, 45:814$821,1988$. 
Pritchard, W.S. Cognitive event-related-potential correlates of schizophrenia. Psychological Bulletin, 1:43-66, 1986.

Roemer, R.A., and Shagass, C. Replication of an evoked potential study of lateralized hemispheric dysfunction in schizophrenics. Biological Psychiatry, 28:275-291, 1990.

Rossi, A.; Stratta, P.; D’Albenzio, L.; Tartaro, A.; Schiazza, G.; di Michele, V.; Bolino, F.: and Casacchia, M. Reduced temporal lobe areas in schizophrenia: Preliminary evidence from a controlled multiplanar magnetic resonance imaging study. Biological Psychiatrv, 27:61-68, 1990.

Roth, W.T., and Cannon, E.H. Some features of the auditory evoked response in schizophrenics. Archives of General Psychiatry, 27:466-471, 1972.

Roth, W.T.; Horvath, T.B.; Pfefferbaum, A.; and Kopell, B.S. Event-related potentials in schizophrenics. Electroencephalography and Clinical Neurophysiology, 48:127-139, 1980.

Smith, M.E.; Halgren, E.; Sokolik, M.; Baudena, P.; Musolino, A.; and Liegeois-Chauvel, C. The intracranial topography of the $\mathrm{P} 3$ event-related potential elicited during auditory oddball. Electroencephalography and Clinical Neurophysiology, 76:235-248, 1990.

Strik, W.K.; Dierks, T.; Franzek, E.; Maurer, K.; and Beckmann, H. Differences in P300amplitudes and topography between cycloid psychosis and schizophrenia in Leonhard's classification. Acta Psychiatrica Scandinavica, 87:179-183, 1993.

Strik, W.K.; Dierks, T.; and Maurer, K. Auditory P300 in schizophrenic patients: Topography and clinical correlations. Proceedings of the Third International Congress on Brain Electromagnetic Topography. Amsterdam: ISBET, 1992. p. 30.

Wood, C.C., and McCarthy, G. A possible frontal lobe contribution to scalp P300. Neuroscience Abstracts, 11:879, 1985. 